\title{
Sistemática y distribución geográfica de Heterachthes flavicornis (Coleoptera: Cerambycidae)
}

\author{
Osvaldo R. Di Iorio
}

Soler 1167, (CP 1615) Grand Bourg, Buenos Aires, Argentina. (megacyllene@yahoo.com.ar)

\begin{abstract}
Systematics and geographical distribution of Heterachthes flavicornis. Revision of 973 specimens of Heterachthes flavicornis (Thomson, 1865), mostly emerged from a same individual of different host plant species, shows that this species has a high phenotypical variation in the color of elytra, antennae and legs. This variability is not related to the geographical distribution or to the host plants. Heterachthes flavicornis sexsignatus (Thomson,1865), and Heterachthes flavicornis bonariensis (Thomson,1865) are considered into the synonymy of Heterachthes flavicornis (Thomson, 1865).
\end{abstract}

KEYWORDS. Coleoptera, Cerambycidae, Heterachthes flavicornis, systematics.

\section{INTRODUCCIÓN}

Thomson (1865) describió tres especies, situadas actualmente en el género Heterachthes Newman, 1840: $H$. flavicornis, $H$. sexsignatus y $H$. bonariensis. En su revisión de Ibiodinini, MARTINS (1970) consideró a las dos segundas como subespecies de la primera, ilustró los diseños elitrales, y dió la distribución geográfica de cada una y de las formas intermedias entre ellas, así como los caracteres que permiten diferenciarlas. Además, MARTINS (1970) colocó a Heterachthes bellus Gounelle, 1909 en sinonimia de $H$. sexsignatus, y a Heterachthes implicatus (Thomson, 1867) en sinonimia de H. bonariensis.

En la presente contribución se analiza y discute la variabilidad presentada en Buenos Aires y en el resto de Argentina, llegándose a la conclusión de que se trata de una sola especie con una gran variabilidad fenotípica, no correlacionada geográficamente ni con sus plantas hospedadoras, y que esta mayor variabilidad no permite separar H. sexsignatus de H. bonariensis.

\section{MATERIAL Y MÉTODOS}

Durante 1994-1995, numerosos ejemplares referidos como Heterachthes flavicornis bonariensis DI IORIO (1996b), fueron criados de varias plantas hospedadoras, todas ellas exóticas respecto de la flora del Espinal periestépico en la provincia de Buenos Aires, Argentina. Posteriormente, numerosos ejemplares fueron también obtenidos de diversas plantas hospedoras cerca de Mar del Plata (Ruta 226, km 22.7), los que, sumados al material anterior y de otras colecciones entomológicas, hacen un total de 973 ejemplares revisados. Los nuevos registros de plantas hospedadoras se señalan con un asterisco precediendo el nombre de cada planta. Abreviaturas de las colecciones examinadas: BERG, Carlos Berg (MACN); GZSM, Gastón Zubaran, San Miguel, Buenos Aires; MACN, Museo Argentino de Ciencias Naturales "Bernardino Rivadavia", Buenos Aires; MMLS, Museo Municipal de Ciencias Naturales "Lorenzo Scaglia", Mar del Plata, Buenos Aires; MZER,
Mateo Zelich, Liebig, Entre Ríos; ODI, Osvaldo Di Iorio, Buenos Aires.

\section{Heterachthes flavicornis (Thomson, 1865)}

Ibidion flavicornis THOMSON, 1865:571.

Heterachthes flavicornis; AuRIVILLIUs, 1912:111.

Heterachthes flavicornis flavicornis; MARTINS, 1970:1261-1265 (redescr.; distr.)

Ibidion sexsignatus Тномson, 1865:572.

Heterachthes sexsignatus; Aurivillius, 1912:111; Martins, 1970:1265-1268 (redescr.; distr.); syn. nov.

Ibidion bonariensis Thomson, 1865:572.

Heterachthes bonariensis: AuRIVILLIUs, 1912:110; ANóNIMO, 1925 lam. 3, fig. 5 (hosp.; como "taladro marrón de los frutales"); BosQ, 1934:338 (distr.; hosp.); 1943:30 (distr.; hosp.); Blanchard, 1939:106, fig. 87d (distr.; hosp.); DirecCión DE Sanidad Vegetal, 1940 (distr.; hosp.); Chiesa Molinari, 1948:177 (hosp.); Bose \& Ruffinelli, 1951:11 (distr.).

Heterachthes flavicornis bonariensis; MARTINS, 1970:1269-1271 (redescr.; distr.); Di IoRIo, 1996b (distr.; hosp.); syn. nov.

Heterachthes bellus Gounelle, 1909: MARTINS, 1970:1265 (sin.). Heterachthes implicatus (Thomson, 1867): MarTins, 1970:1269 ( $\sin$.).

Distribución geográfica. BRASIL: Bahia, Espírito Santo, Rio de Janeiro, Minas Gerais, São Paulo, Paraná, Santa Catarina, Rio Grande do Sul, Goiás, Mato Grosso do Sul; PARAGUAY: Caaguazú; URUGUAY: Colonia, Rivera, Montevideo; ARGENTINA: Misiones, Buenos Aires, Entre Ríos.

Material examinado. ARGENTINA, Misiones: Iguazú, 1 ex., X.1927 (MACN) [Martins det. 1968, Heterachthes flavicornis interm. "2" (Th.)"]; Entre Ríos: Liebig, 1 ex., Zelich col. (MZER); Buenos Aires: sin localidad, 1 ex. (MACN) [Martins det. 1966]; Otamendi, Estación I.N.T.A., 1 ex. (ODI); Tigre, 1 ex., "35559" (MACN); Delta, Cruz Colorada, 1 ex., Daguerre col. (MACN); Delta (arroyo Caraguatá: Bachmann, com. pers.), Bachmann col., 1 ex., 2.XI.1945 (MACN), 1 ex., 18.XI.1945 (MACN); Martinez, 1 ex., "40", Frers col. (MACN); 1 ex., "41" (MACN); Olivos, 1 ex., XI.1945, Aloisi col. (MACN); Vicente Lopez, 2 exs., XI.1944 (MACN); 1 ex., 4.XII.1949 (MACN); 1 ex., II (o XI ?).1942 (MACN) [Martins det. 1968]; 1 ex., II (o XI ?).1944 (MACN); Capital Federal, 1 ex., 20.XII.1946, Bachmann col. (MACN); Villa Devoto, 1 ex., 9.XI.1928, Matus col. (ODI) [Martins det. 1962]; 1 ex., 15.XI.1926, Mühn col. (ODI); 1 ex., 30.XI.1927, Mühn col. (ODI) [Bruch C. det.]; 1 ex., 15.XI.1930, 
Fincheira col. (ODI); 1 ex., XI.1926, Bridarolli col. (ODI) [Martins det. 1962]; 1 ex., 1.IV.1926, Bridarolli col. (ODI) [Martins det. 1962]; Adrogué, 1 ex., I.1921 (MACN) [Martins det. 1968]; La Plata, 3 exs., 20.XI.1920 (BERG); Mar del Plata, 1 ex., 1.I.1981, M.M. Ceccotti col. (MMLS); 5 exs., 21.XII.1982, Farina col. (MMLS); 1 ex., 1.I.1998, H. Cordo col. (ODI); 1 ex., 2.XII.1998, Farina col. (MMLS).

Plantas hospedadoras, localidades y ejemplares emergidos

*Anadenanthera colubrina var. cebil (Griseb.) Alts. (Mimosaceae). Buenos Aires: Grand Bourg, 4 exs., 3.VII.1994 (larvas), Di Iorio col. (ODI) (infestación secundaria; ramas mantenidas a la intemperie, con larvas de Oncideres germari Thomson).

*Acacia melanoxylon R. Brown (Mimosaceae). Buenos Aires: Ruta 226, Km 22.7, 22.VIII.2001 (larvas), Di Iorio \& Farina col., 72 exs. (ODI, MMLS) (primera generación), 9 exs. (MMLS) (segunda generación).

* Carya illinoensis K. Koch (Juglandaceae). Buenos Aires: Grand Bourg, 1 ex., 12.VII.2002 (larva), Di Iorio col. (ODI)

Feijoa sellowiana O. Berg (Myrtaceae). Buenos Aires: Merlo, 14 exs., 10.IX.1995 (larvas), Di Iorio col. (ODI).

Ficus carica Linn. (Moraceae). Buenos Aires: Ruta 226, Km 22.7, 30.VI.2001 (larvas), Di Iorio \& Farina col., 68 exs. (ODI, MMLS); mismos datos, 29.V.2002 (larvas), 68 exs. (ODI, MMLS).

Laurus nobilis L. (Lauraceae). Buenos Aires: Merlo, 19 exs., 20.XI.1994 (larvas), Di Iorio col. (ODI).

Maclura pomifera (Raf.) Schneid. (Moraceae). Buenos Aires: Partido de Pilar, Villa Rosa, vías F.C.G.B. y río Luján (larva), 1 ex., Di Iorio col. (ODI).

* Melia azedarach L. (Meliaceae). Buenos Aires: San Miguel, 5 exs., 24.XII.2002 (larvas), Zubaran col. (GZSM).

Morus alba L. (Moraceae). Buenos Aires: Merlo, 38 exs., 20.VIII.1994 (larvas), Di Iorio col. (ODI).

Morus nigra L. (Moraceae). Buenos Aires: Merlo, 51 exs., 10.IX.1995 (larvas), Di Iorio col. (ODI).

Persea americana L. (Lauraceae). Buenos Aires: Grand Bourg, 33 exs., 3.VII.1994 (larvas), Di Iorio col. (ODI).

*Pinus insignis L. (Pinaceae). Buenos Aires: Ruta 226, Km 22.7, 30.VI.2001 (larvas), Di Iorio \& Farina col., 39 exs. (ODI, MMLS).

Populus sp. (Salicaceae). Buenos Aires: San Miguel, 5 exs., 24.XII.2002 (larvas), Zubaran col. (GZSM).

Prunus domestica L. (Rosaceae). Buenos Aires: Merlo, 33 exs., 13.XI.1994 (larvas), Di Iorio col. (ODI); Ruta 226, Km 22.7, 18 exs., 30.VI.2001 (larvas), Di Iorio \& Farina col. (ODI); 109 exs., 29.V.2002 (larvas) (ODI, MMLS)

Prunus persica (L.) Batsch. (Rosaceae). Buenos Aires: Grand Bourg, 327 exs., 3.VII.1994 (larvas), Di Iorio col. (ODI).

*Quercus palustris Muench. (Fagaceae). Buenos Aires: Ruta 226, Km 22.7, 2 exs., 30.VI.2001 (larvas), ramas secas por larvas de Praxithea derourei (Chabrillac, 1858), Di Iorio \& Farina col. (ODI).

Salix sp. (Salicaceae). Buenos Aires: Ruta 226, Km 22.7, 11 exs., 30.VI.2001 (larvas), Di Iorio \& Farina col.
(ODI, MMLS).

Vitis vinifera L. (Vitaceae). Buenos Aires: Merlo, 16 exs., 13.XI.1994 (larvas), Di Iorio col. (ODI).

Discusión taxonómica. En realidad se trata de una sola especie, altamente variable en su área de distribución, lo que se evidencia por los siguientes hechos: 1) la presencia de ejemplares intermedios entre $H$.f.flavicornis y $H . f$. bonariensis en el área de distribución de $H . f$. flavicornis (MARTINS, 1970), cuando estos dos fenotipos están respectivamente en los extremos norte y sur del área de distribución; 2) la presencia de H.f. sexsignatus (fig. 5) en un lote de ejemplares intermedios entre este fenotipo y $H$. f. bonariensis (figs. 9-10, 13, 18) en una localidad (Santa Catarina, Nova Teutonia) donde solo deberían encontrarse los fenotipos intermedios (MARTINS, 1970); 3 ) ejemplares intermedios entre $H$.f. sexsignatus y $H$.f. bonariensis y ejemplares de $H$.f. bonariensis en una misma localidad (Montevideo); 4) la emergencia de un mismo individuo de planta hospedadora y entre localidades diferentes (Grand Bourg, Merlo, Ruta 226) de ejemplares correspondientes a H.f. sexsignatus (fig. 5), H.f. bonariensis (figs. 16-17, 19), fenotipos intermedios descriptos por MARTINS (1970) (figs. 9-10, 13, 18) y fenotipos no descriptos anteriormente (figs. 1-4, 6-8, 11 12, 14-15, 20-26).

Ningún ejemplar de Buenos Aires lleva el ápice de los élitros oscuro como ilustrado por MARTINS (1970: figs. 638-641) para H.f.flavicornis. En cambio, todos tienen el ápice de los élitros de color claro, aunque tienen el área adyacente al escutelo también oscura (figs. 1-4). Solo dos de los diseños elitrales de los ejemplares de Buenos Aires (figs. 11-12) son similares al diseño de $H . f$. flavicornis, con el ápice de los élitros claro y el área adyacente al escutelo también clara (MARTINS, 1970: fig. 642), pero la diferencia radical es que en estos ejemplares de Buenos Aires el área humeral también es clara. En todos los diseños elitrales de Buenos Aires (figs. 1-4, 6-8, 11-12, 14-15, 20-26), el área humeral también es clara, y se mantiene este color aunque reducido a una pequeña extensión aún en los ejemplares mas oscuros (figs. 1-4).

De esta manera, H.f.flavicornis, H.f. bonariensis y $H$.f. sexsignatus quedan incluídos dentro de una serie continua en la cual no pueden establecerse límites netos entre uno y otro fenotipo (figs. 1-26), y que van desde formas predominantemente oscuras (figs. 1-20) hasta otras predominantemente claras, casi amarillas (figs. 21-26). Los diseños menos representados son los extremos de la serie, es decir, los mas oscuros (figs. 1-4) y los mas claros (figs. 21-26). Las formas oscuras fueron mas frecuentes en Laurus nobilis, aunque no existe una clara dependencia entre el diseño elitral y las respectivas plantas hospedadoras. El color de las patas y antenas tampoco está asociado a ningún diseño elitral en particular.

De acuerdo a lo anterior, y para simplificar la nomenclatura existente, se propone que las tres subespecies nombradas y las formas intermedias entre ellas se reduzcan a una sola especie válida, Heterachthes flavicornis, de amplia distribución y variación fenotípica.

En general, Heterachthes flavicornis sigue uno de 

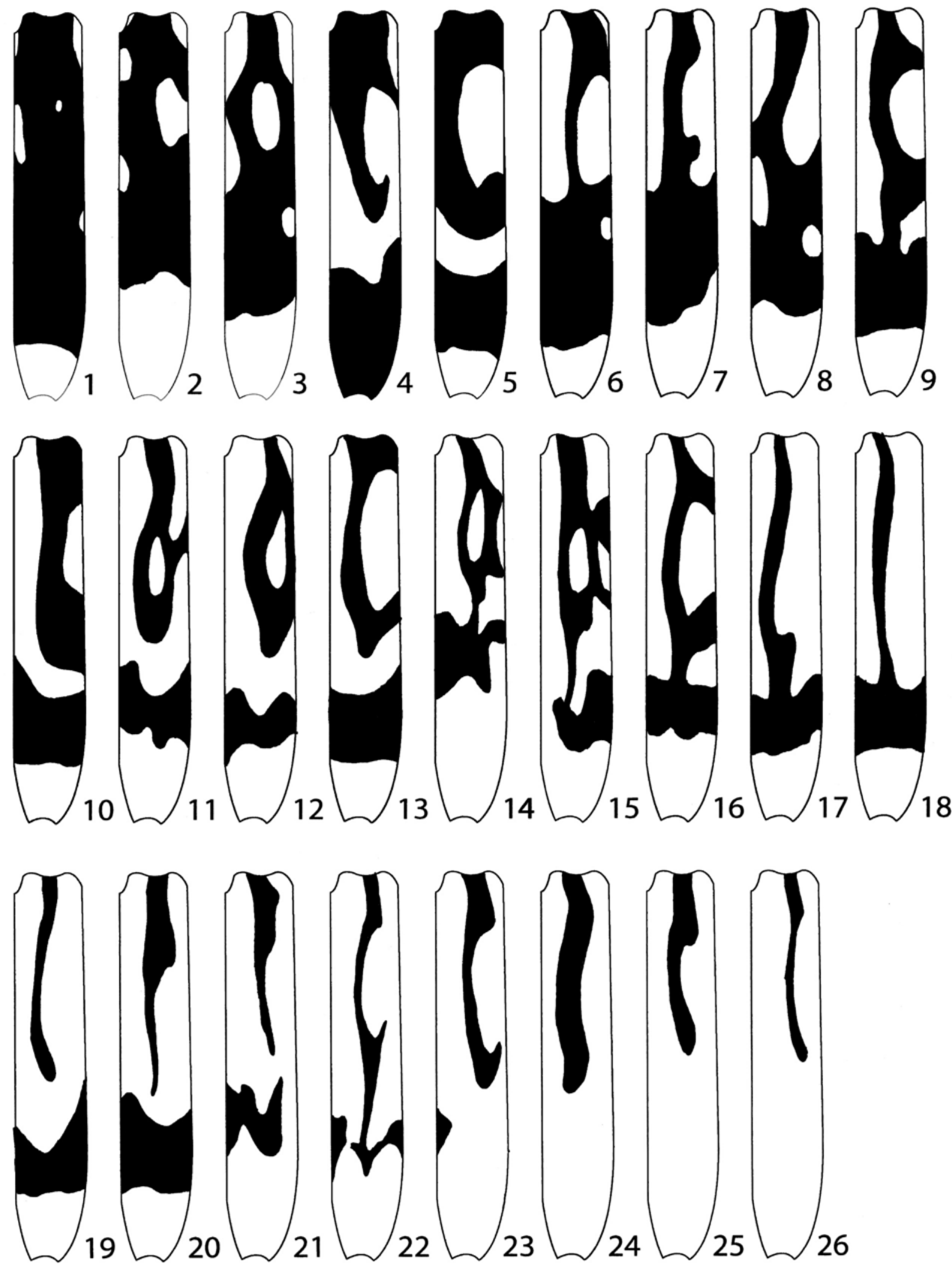

Figs. 1-26, diseños elitrales: 1-4; 6-8; 11-12; 14-15; 20-26, ejemplares obtenidos de diversas plantas en Buenos Aires (Grand Bourg, Merlo, Ruta 226); 5, H.f. sexsignatus (Martins, 1970); 9-10, 13, 18, ejemplares intermedios entre H. f. sexsignatus y H. f. bonariensi. (MARTins, 1970). 
los patrones geográficos de distribución observados en otras especies de Cerambycidae, al este del Chaco sensu stricto, desde el este de Brasil hasta el este del Paraguay, y el este de la Argentina y Uruguay (Di IORIO, 1996a). La distribución en la Argentina está restringida a la región mesopotámica (Misiones, Entre Ríos), aunque falta encontrarlo aún en Corrientes, y alcanza su límite austral en Buenos Aires, Partido de General Pueyrredón (Mar del Plata; Ruta 226, km 22.7), dentro de los límites del Espinal periestépico (LEwIS \& Collantes, 1973). En Buenos Aires, la adaptación a numerosas plantas hospedadoras exóticas posiblemente ha contribuído a una mayor dispersión geográfica ocurrida en tiempos históricos, durante la colonización española.

\section{REFERENCIAS BIBLIOGRÁFICAS}

Anónimo. 1925. Principales insectos que dañan los frutales de hoja caduca. Ministerio de Agricultura de la Nación, Sección Propaganda e Informes, Circular, Buenos Aires, (528):1-38.

Aurivillius, C. 1912. Coleopterorum Catalogus, pars 39, Cerambycidae: Cerambycinae. Berlín, W. Junk. 574p.

Blanchard, E. E. 1939. Los animales enemigos de la fruticultura argentina y los medios de combatirlos. Ministerio de Agricultura de la Nación, Publicación Miscelánea, Buenos Aires, 58:1-192.

Bosq, J. M. 1934. Primera lista de los coleópteros de la
República Argentina dañinos a la agricultura. Boletín del Ministerio de Agricultura de la Nación, Buenos Aires, 36(4):313-346.

1943. Segunda lista de coleópteros de la República Argentina dañinos a la agricultura. Ministerio de Agricultura de la Nación, Dirección de Sanidad Vegetal, División de Zoología Agrícola, Buenos Aires, 80p.

BosQ, J. M. \& Ruffinelui, A. 1951. Notas para el catálogo de Cerambícidos del Uruguay. Comunicaciones Zoologicas del Museo de Historia Natural de Montevideo, Montevideo, 3(62):1-32.

Chiesa Molinari, O. 1948. Las plagas de la agricultura. Manual práctico de procedimientos para combatirlas. El Ateneo, Buenos Aires. 497p.

Di Iorio, O. R. 1996a. Plantas hospedadoras de Cerambycidae (Coleoptera) del noroeste y centro de Argentina. Revista de Biología Tropical, Turrialba, 44(3):149-158.

1996b. Plantas hospedadoras de Cerambycidae (Coleoptera) en el Espinal y en la provincia de Buenos Aires, Argentina. Revista de Biología Tropical, Turrialba, 44(3):159-165.

Dirección de Sanidad Vegetal. 1940. Boletín Informativo, Buenos Aires, 4(13):1-52.

Lewis, J. P. \& Collantes, M. B. 1973. El Espinal periestépico. Ciencia e Investigación, Buenos Aires, 29:360-377.

Martins, U. R. 1970. Monografia da tribo Ibidionini (Coleoptera, Cerambycinae). Parte V. Arquivos de Zoologia, São Paulo, 16(5):1151-1342.

Thomson, J. 1865. Diagnoses d'espèces nouvelles qui seront décrites dans 1'appendix du systema cerambycidarum. Mémoires de la Société Royale des Sciences de Liège, Liège, 19:541-578 